Voix et Images

volxetimages

\title{
La littérature québécoise dans le cadre des études francophones en Autriche
}

\section{Fritz Peter Kirsch}

Volume 26, numéro 2 (77), hiver 2001

Denise Desautels

URI : https://id.erudit.org/iderudit/201552ar

DOI : https://doi.org/10.7202/201552ar

Aller au sommaire du numéro

Éditeur(s)

Université du Québec à Montréal

ISSN

0318-9201 (imprimé)

1705-933X (numérique)

Découvrir la revue

Citer cet article

Kirsch, F. P. (2001). La littérature québécoise dans le cadre des études francophones en Autriche. Voix et Images, 26(2), 422-426.

https://doi.org/10.7202/201552ar d'utilisation que vous pouvez consulter en ligne.

https://apropos.erudit.org/fr/usagers/politique-dutilisation/ 


\title{
La littérature québécoise dans le cadre des études francophones en Autriche
}

\author{
Fritz Peter Kirsch, Université de Vienne
}

À l'heure actuelle, l'Autriche ne possède pas de centres d'études consacrés exclusivement aux cultures de langue française en Amérique du Nord, tels qu'on en trouve en Allemagne (Trèves, Dresde). Pourtant les littératures québécoise et francocanadienne occupent une place importante dans trois des cinq villes universitaires du pays (Vienne, Innsbruck et Graz). Ce fait est dû à un changement profond dans la conception et dans les structures des études romanes (Romanische Philologie, Romanistik), cette discipline spécifique aux pays de langue allemande dont les origines remontent au $\mathrm{xxx}^{\mathrm{e}}$ siècle romantique et qui embrasse, en principe, la totalité des cultures néolatines. Si l'étudiant viennois en philologie romane, dans les années soixante, s'occupait encore presque exclusivement de l'histoire de la langue et de la littérature françaises (hexagonales) et italiennes (les domaines espagnols et roumains faisant plutôt figure de parents pauvres), au cours des décennies suivantes, cet étudiant était confronté avec l'avancée lente mais irrésistible de la "Nou- 
velle Romania " comprenant le domaine hispano-américain, la lusophonie brésilienne/africaine et la francophonie. Aujourd'hui, cette évolution conduit à des résultats concrets. Après une période d'apprentissage et de découvertes, il fallait bien opérer des choix pour faire face à l'ampleur des nouvelles littératures que notre discipline voulait désormais s'annexer. D'une façon générale, on peut dire que les études de la littérature de langue française en Amérique du Nord ont profité de ce travail de décantation. Dans les départements d'études romanes des trois universités mentionnées, la littérature québécoise occupe aujourd'hui une place importante, en se situant toutefois dans un contexte "francophone" où la France continue à jouer un rôle primordial. Cette intégration, comme on le verra, n'a nullement empêché l'émergence de spécialistes se consacrant à la publication de monographies ou d'articles sur des sujets québécois.

Dans la trajectoire qu'on vient d'esquisser, la création d'une Association internationale des Études francophones d'Europe Centre-Orientale (AEFECO), au début des années quatre-vingt-dix, a joué un rôle déterminant. Après la chute du rideau de fer, l'AEFECO est née des efforts communs de quelques romanistes hongrois et autrichiens visant à rapprocher les francophones des pays ex-communistes de leurs confrères occidentaux. Cette coopération, impliquant d'abord les Universités de Pécs et de Vienne, a bientôt permis la rencontre de partenaires dans plusieurs pays européens, en Afrique et en Amérique du Nord. Elle s'est consolidée grâce à des colloques (Pécs
1992, Vienne 1995, Leipzig 1998), et a permis la création d'une revue, les Cabiers francophones d'Europe Centre-Orientale (dirigée par Arpád Vigh et Fritz Peter Kirsch), paraissant une fois l'an et faisant graviter les contributions de chaque numéro autour d'un thème ${ }^{1}$.

Des universitaires autrichiens se sont également engagés dans le cadre de l'Association des Études canadiennes dans les pays de langue allemande (GKS). Madame Ursula Moser-Mathis, professeure au Département d'Études romanes de l'Université d'Innsbruck, a été la viceprésidente de cette association entre 1993 et 1995, et présidente de 1995 à 1997. Depuis 1997, Fritz Peter Kirsch (Vienne) dirige la section d'études francophones au sein de la GKS.

Les recherches concernant l'Amérique francophone ont également profité de la naissance récente de centres d'études canadiennes au sein des trois universités mentionnées. Le premier pas a été fait par Innsbruck, où un tel centre a vu le jour en 1997 grâce à l'initiative de madame MoserMathis qui en assure la direction. Cependant, avant la mise en place de cette institution d'orientation générale, Ursula Moser-Mathis avait déjà créé un Centre d'étude de la chanson québécoise (1994) dont le but déclaré était de recueillir tous les documents sonores et imprimés disponibles dans le domaine de la "musique à texte" franco-canadienne. Ce centre dispose actuellement d'une phonothèque de plus de 10,000 titres (disques, cassettes) mémorisés dans une banque de données et accessibles aux chercheurs grâce à un studio d'enregistrement attenant aux archives. En 1993, la directrice a organisé 
un colloque international sur la chanson (avec une forte participation québécoise). Quatre ans plus tard, madame Moser-Mathis a publié une discographie dans la série Canadiana oenipontana. Mentionnons, également, qu'au centre d'Innsbruck, des spécialistes de la littérature québécoise ont enseigné en tant que professeurs invités (par exemple Sherry Simon, Pierre L'Hérault).

L'année 1998 a vu la naissance d'un Centre d'études canadiennes à Vienne, dans le cadre stimulant du nouveau campus universitaire, en plein centre-ville, sur la base d'une coopération entre le Département d'études anglaises (Waldemar Zacharasiewicz) et le Département d'études romanes (Fritz Peter Kirsch). Dans le programme scientifique du colloque grâce auquel on a fêté l'inauguration de ce centre ${ }^{2}$, les études francophones ont été représentées par Yolande Grisé (historienne de la civilisation, Ottawa) et Lothar Wolf (linguiste, Augsburg). Lors d'un deuxième colloque qui a eu lieu en avril $1999^{3}$, des sujets concernant l'Amérique francophone ont été traités par Sylvie Dubois (Bâton Rouge), Eva Martonyi (Budapest), Klaus Ertler (Graz) et Margareta Gyurcsik (Timisoarã). Naturellement, les centres de Vienne et d'Innsbruck ne se font pas prier lorsque se présente une occasion d'inviter d'illustres conférenciers québécois séjournant en Europe $^{4}$. Ces centres s'efforcent de coopérer avec des institutions semblables en Hongrie où une équipe d'études canadiennes vient de se constituer à l'Université catholique de Budapest/Piliscsaba. À l'automne 1999, grâce à l'initiative du romaniste KlausDieter Ertler, un Centre d'études canadiennes pourvu d'un Centre de docu- mentation a ouvert ses portes à Graz. Ce centre, qui privilégie les recherches portant sur la littérature coloniale de la Nouvelle-France ainsi que sur le film francophone au Québec, a été inauguré par un colloque intitulé "Canada 2000 : Identité et transformation ${ }^{5}$. Klaus-Dieter Ertler se propose de coopérer plus étroitement avec les canadianistes des universités slovènes (Maribor, Ljubljana).

À Vienne, la situation des études francophones se caractérise par le fait que, depuis 1993, un poste de professeur invité est réservé aux cultures maghrébines et subsahariennes. Madame Zohra Bouchentouf-Siagh, sociolinguiste algérienne, occupe actuellement ce poste. Fritz Peter Kirsch, avant l'arrivée de madame Bouchetouf-Siagh, seul professeur du département proposant des cours et des séminaires sur les littératures de la francophonie d'outre-mer, se consacre actuellement, en premier lieu, à la littérature de langue française au Canada en faisant alterner des cycles québécois avec des cycles de littérature française "hexagonale". Du côté de la linguistique, le professeur Georg Kremnitz privilégie les études portant sur les contacts et les conflits sociolinguistiques et socioculturels, notamment dans le domaine des études antillaises. Autour des professeures et professeurs mentionnés, de nouvelles générations se manifestent. Des thèses ont été soutenues sur la conscience linguistique des Suisses romands, les variétés du français dans les îles de la Manche, les structures romanesques chez Mohammed Dib, le thème de l'uÉtrangère " chez les romanciers du Québec, les aspects traditionalistes de l'œuvre narrative de Gabrielle Roy, etc. Une 
vingtaine de mémoires sur des sujets québécois, maghrebins et subsahariens ont été déposés.

À Innsbruck, les cycles de cours proposés par Ursula Moser-Mathis ont assuré la continuité de l'enseignement des littératures francophones depuis la fin des années quatre-vingt. Birgit Mertz-Baumgartner a reçu le "Prix d'Excellence du Québec "pour sa thèse sur'le monologue québécois, publiée en 1997 aux éditions Wissner à Augsburg ${ }^{6}$. Dernièrement, une autre étudiante de madame MoserMathis, Beate Burtscher-Bechter, a profité de la première co-tutelle de thèse entre la Sorbonne (Paris IV) et l'Autriche. La soutenance de cette thèse sur le roman policier algérien, devant le jury présidé par madame Moser-Mathis et comptant parmi ses membres Jacques Chévrier, Guy Dugas et Robert Jouanny, a eu lieu en 1998. Étant donné que le Maghreb représente, à côté du Québec, le deuxième centre d'intérêt des collègues d'Innsbruck, ces dernières ont organisé, au début de l'année 2000 , une "Semaine algérienne" dont le programme a été marqué par la présence d'écrivains et d'universitaires maghrébins.

Au sein du Département d'Études romanes de l'Université de Graz, les études francophones sont représentées surtout par Klaus-Dieter Ertler dont la monumentale thèse d'habilitation sur le roman québécois de l'Entre-deux-guerres a été soutenue en juin $1999^{7}$. Ertler donne régulièrement des cours de travaux pratiques sur les littératures du Canada francophone. La "Petite bistoire du roman franco-canadien", qu'il vient de publier chez un éditeur allemand ${ }^{8}$, constitue un important pas en avant sur le plan de la réception de la littérature québécoise dans les pays de langue allemande où, à l'heure actuelle, les écrivains "francophones" du Maghreb ou de l'Afrique noire ont été beaucoup plus traduits que leurs confrères d'Amérique du Nord.

Il est normal que les spécialistes mentionnés prennent part, de temps à autre, à l'enseignement auprès d'autres universités. Fritz Peter Kirsch, par exemple, a enseigné les littératures québécoise, maghrébine et subsaharienne à Pécs, Paris (Université Paris III), Nice et Bucarest. Peu à peu, d'ailleurs, l'enseignement secondaire autrichien s'est ouvert au "fait francophone". Au cours de la dernière décennie, Ursula MoserMathis et Fritz Peter Kirsch ont dirigé plusieurs séminaires ou stages de recyclage pour des professeurs de lycée, en orientant ces derniers vers des thématiques québécoises.

Faisons état, pour finir, de quelques travaux en cours. L'orientation des trois chercheurs qui ont été souvent mentionnés au cours de ce survol se distingue par le fait que madame Moser-Mathis et monsieur Ertler sont de "vrais" spécialistes de la littérature québécoise alors que Fritz Peter Kirsch, après avoir travaillé sur les clivages interculturels en France, fait du comparatisme "francophonisant " en s'appuyant sur les thèses sociohistoriques de Norbert Elias. Actuellement, Ursula Moser-Mathis, après avoir publié plusieurs études sur des sujets québécois ( littératures migrantes", littérature féminine), prépare une monographie consacrée à Dany Laferrière. Klaus-Dieter Ertler travaille actuellement sur des corpus littéraires en faisant valoir une approche théorique originale qui consiste à envisager 
l'histoire littéraire de l'Amérique francophone à partir du systémisme. Fritz Peter Kirsch a publié un livre portant sur certaines analogies entre les littératures de la francophonie et la littérature occitane ${ }^{9}$ aux Presses universitaires de Bordeaux, l'automne dernier.

1. Voici les titres des numéros parus : «Mots du Québec * (1991), : Cultures en conflit • (1992), - L'Enseignement de la francophonie. (1993), - La Suisse ouverte: Nicolas Bouvier. (1994), -Y a-t-il un dialogue interculturel dans les pays francophones? (1995), *Belgique francophone: Quelques façons de dire les Mixités * (1997), *Anciens Canadiens et nouveaux Québécois. Hommage à Gaston Miron (1998), - Unité et diversité des écritures francophones. Quels défis pour cette fin de siècle?. (sous presse)

2. Canada/Europe: chances et malaises de l'interculturalité ", Vienne, octobre 1998.

3. Perspectives interculturelles: le Canada et les États-Unis .

4. C'est ainsi qu'on a pu entendre Maurice Lemire, Gilles Dorión, Nicole Brossard, JeanFrançois Chassay, Danielle Fournier, Micheline Cambron, Marco Micone...

5. Des contributions consacrées à des sujets québécois ont été présentées par Peter Klaus (Berlin), Ljiljana Matic (Novi Sad), Alessandra Ferraro (Udine).

6. Monologues québécois oder Geschichten eines/Monsieur qui parle tout seul. Standortbestimmung einer Gattung am Rande, Augsburg, Wissner, 1997.

7. Erzäblte Ideologie versus Ideologische Erzäblung in der frankokanadiscben Literatur der 30er Jabre (sous presse).

8. Klaus-Dieter Ertler, Kleine Geschichte des frankokanadischen Romans, Tübingen, Narr, 2000.

9. Fritz Peter Kirsch, Ecrivains au carrefour des cultures. Études de littérature occitane, francaise et "francopbone. 